\title{
Rilievo in tempo reale di difetti superficiali su corpi in movimento a velocità elevata con ultrasuoni senza contatto
}

\author{
Real-time non-contact ultrasonic detection \\ of surface defects on objects moving at high speed
}

\author{
D. Cerniglia, V. Nigrelli, A. Mancuso, A. Alberti \\ Dipartimento di Ingegneria Chimica, Gestionale, Informatica, Meccanica, Università degli Studi di Palermo, viale delle Scienze, \\ 90128 Palermo, Italy \\ vincenzo.nigrelli@unipa.it
}

\begin{abstract}
The detection of surface defects during periodic inspection is important because, usually, the stresses are higher at the surface, thus affecting the growth of the discontinuity. Surface defects can be detected using some traditional non-destructive testing methods, such as eddy current, dye penetrant, magnetic particle and ultrasonic. Some of these methods can be used only in static conditions; the others have limitations for the dynamic inspection.

The recent progress in the field of non-contact ultrasonic sensors has led us to develop a simple system for the real-time inspection of moving bodies at a high speed. The paper presents the possibility of using, with the developed system, two methodologies, based on non-contact ultrasound, to detect surface defects on objects moving at $100 \mathrm{~km} / \mathrm{h}$, so that in service inspection is possible. A methodology, based on laser and air-coupled sensors, uses the advantages of laser-generated surface waves; the analysis is done on the reflected wave, created by the interaction of the surface wave with the defect. The inspected surface layer can be selected from the wavelength of the surface wave. The other methodology, based on air-coupled sensors, uses the disadvantages of the ultrasound transmission at the air/metal interface; the analysis is done on the diffraction of the wave reflected from the surface. The execution of the inspection is simple with both techniques. The experimental results indicate a good efficiency of the two methodologies proposed for the real-time detection of surface defects on objects moving at high speed.
\end{abstract}

SOMMARIO. Il rilievo di difetti superficiali durante le ispezioni periodiche è importante poiché in genere le sollecitazioni sono maggiori in superficie e possono accelerare la crescita delle discontinuità. I difetti superficiali possono essere rilevati utilizzando alcuni metodi tradizionali di controllo non distruttivo, quali correnti indotte, liquidi penetranti, polveri magnetiche ed ultrasuoni. Alcuni tra questi metodi possono essere usati solo in condizioni statiche; gli altri presentano delle limitazioni per l'ispezione dinamica. I progressi recenti nel campo dei sensori ultrasonori senza contatto ci hanno permesso di sviluppare un sistema semplice per l'ispezione in tempo reale di corpi in movimento a velocità elevata. Nel lavoro viene presentata la possibilità di usare, col sistema sviluppato, due metodologie, basate sugli ultrasuoni generati e ricevuti senza contatto con la struttura, per rilevare difetti superficiali su corpi in movimento a $100 \mathrm{~km} / \mathrm{h}$, così da poter effettuare l'ispezione anche in servizio. Una metodologia, basata sull'uso di laser e trasduttori senza contatto, utilizza i vantaggi delle onde superficiali generate con il laser; l'analisi viene fatta sull'onda riflessa, creata dall'interazione dell'onda superficiale con il difetto. Lo spessore superficiale ispezionato è selezionabile dalla lunghezza d'onda dell'onda 
superficiale generata. L'altra metodologia, basata sull'uso di trasduttori senza contatto, sfrutta gli svantaggi della trasmissione degli ultrasuoni all'interfaccia aria/metallo; l'analisi viene fatta sulla diffrazione dell'onda riflessa dalla superficie. L'esecuzione delle ispezioni risulta semplice con entrambe le tecniche. I risultati sperimentali indicano una buona efficienza delle due metodologie proposte per il rilievo, in tempo reale, di difetti superficiali su corpi in movimento ad alta velocità.

Parole Chiave. Ultrasuoni; Laser; Sensori senza contatto; Difetti superficiali; Ispezione dinamica.

\section{INTRODUZIONE}

I 1 rilievo di difetti superficiali durante le ispezioni periodiche è importante poiché in genere le sollecitazioni sono maggiori in superficie e possono accelerare la crescita delle discontinuità. I difetti superficiali possono essere rilevati utilizzando alcuni metodi tradizionali di controllo non distruttivo, quali correnti indotte, liquidi penetranti, polveri magnetiche ed ultrasuoni. Alcuni tra questi metodi possono essere usati solo in condizioni statiche; gli altri presentano delle limitazioni per l'ispezione dinamica. Le tecniche ad ultrasuoni in cui la trasmissione di energia nel materiale avviene tramite contatto, nonostante siano caratterizzate da affidabilità e semplicità, limitano notevolmente la velocità di ispezione su oggetti in movimento ed anche il rilievo di difetti superficiali, poiché i segnali di eco dei primi millimetri di superficie non sono visibili. Inoltre, in generale, l'ispezione è influenzata dalle condizioni di contatto quali superficie del pezzo in esame, pressione della sonda, mezzo di accoppiamento ed area di contatto.

Negli ultimi decenni l'interesse è stato focalizzato verso metodi e sensori non a contatto poiché consentono di superare questi limiti. In particolare, grazie ai progressi su nuovi materiali per dispositivi acustici e su nuove tecnologie di fabbricazione, si è avuto un crescente sviluppo di nuove sonde [1]. Con aria come mezzo di accoppiamento, la distanza dei trasduttori dalla superficie dei corpi da ispezionare dipende dalla frequenza utilizzata. Inoltre, poiché l'attenuazione delle onde varia con il quadrato della frequenza, si ha un limite alle frequenze rilevabili. Ł̇ infatti impossibile ricevere onde con alta frequenza (maggiore di $5 \mathrm{MHz}$ ) senza portare il trasduttore quasi a contatto con la superficie. Un altro limite principale associato all'impiego dei trasduttori senza contatto è la grande differenza di impedenza acustica all'interfaccia aria/metallo. L'onda riflessa dal metallo (per esempio alluminio) è pari al 99.99\% dell'energia incidente; mentre quella che arriva al trasduttore, dopo aver attraversato metallo ed aria, è prossima al 10-6\%. La combinazione del laser per generare e del sensore senza contatto per ricevere è più efficiente poiché gli ultrasuoni sono prodotti direttamente nel metallo. Eliminando una interfaccia aria/alluminio, l'energia che arriva al trasduttore è pari allo $0.01 \%$. La generazione di onde acustiche tramite laser è ormai ben caratterizzata [2-7]. La propagazione di onde generate tramite laser nei metalli, la loro interazione con i difetti e la trasmissione in aria sono state studiate tramite simulazione numerica in [8,9]. L'uso del laser nei sistemi di controllo ad ultrasuoni mostra anche un interesse crescente nel settore industriale poiché consente l'ispezione remota ed automatizzata, realizzabile su corpi in movimento.

I progressi recenti nel campo dei sensori ultrasonori senza contatto ci hanno permesso di sviluppare un sistema semplice per l'ispezione in tempo reale di corpi in movimento a velocità elevata. Nel lavoro viene presentata la possibilità di usare, col sistema sviluppato, due metodologie per il rilievo di difetti superficiali, in tempo reale, su componenti in movimento alla velocità di $100 \mathrm{~km} / \mathrm{h}$. La prima usa i vantaggi delle onde superficiali generate con il laser [10-11] ed analizza l'onda riflessa, creata dall'interazione dell'onda superficiale con il difetto. La seconda sfrutta gli svantaggi della trasmissione degli ultrasuoni all'interfaccia aria/metallo ed analizza la diffrazione dell'onda riflessa dalla superficie. Il caso esaminato è quello in cui i sensori sono stazionari e il corpo ruota ad elevata velocità; caso per esempio di una stazione di ispezione per le ruote ferroviarie. Questo lavoro è stato motivato da precedenti studi relativi ad onde ultrasonore per l'ispezione dinamica di rotaie e ruote ferroviarie [12-14].

\section{PRove E RISUltati SPERIMENTALI}

$\mathrm{P}$ er l'esecuzione delle prove sono stati utilizzati due dischi in alluminio con diametro esterno $201.2 \mathrm{~mm}$ e spessore 15 $\mathrm{mm}$ : uno integro e l'altro con un difetto superficiale passante, largo $0.5 \mathrm{~mm}$ e profondo $2 \mathrm{~mm}$. I dischi, calettati sull'albero di un motore elettrico, sono stati messi in rotazione con una velocità angolare pari a $2800 \mathrm{giri} / \mathrm{min}$ (velocità periferica pari a $106 \mathrm{~km} / \mathrm{h}$ ). Per il rilievo del difetto sono state usate due diverse metodologie. La prima utilizza, 
in un'unica ispezione, onde superficiali che si propagano in direzione circonferenziale; la seconda realizza un'ispezione continua sull'intera circonferenza, lungo la generatrice del disco.

Il primo setup sperimentale è mostrato in Fig. 1. Le onde superficiali di Rayleigh sono generate tramite un laser pulsato e acquisite tramite due trasduttori piezoelettrici. Le onde superficiali si propagano solo nello strato superficiale dei solidi, seguendone il profilo, e penetrano nel materiale per una profondità pari alla lunghezza d'onda, in relazione quindi alla frequenza di ispezione utilizzata [2].

L'ampiezza dello spostamento delle particelle decresce dalla superficie verso l'interno del mezzo. Il laser utilizzato opera a $1064 \mathrm{~nm}$ con un'energia massima di $900 \mathrm{~mJ}$, frequenza $10 \mathrm{~Hz}$ e durata dell'impulso di $5 \mathrm{nsec}$. I sensori registrano un segnale proporzionale alla pressione acustica, che è la deviazione locale della pressione rispetto a quella ambiente causata dall'onda ultrasonora. Hanno una frequenza nominale di $1.5 \mathrm{MHz}$, larghezza di banda (a -6 dB) $240 \mathrm{kHz}$ e diametro 12 $\mathrm{mm}$. La distanza di ciascun trasduttore dalla superficie del disco è $5.5 \mathrm{~mm}$. I sensori hanno una disposizione simmetrica (Fig. 1) rispetto al piano su cui è il fascio laser, con $\theta_{1}=\theta_{2}=\theta$. Si è assunto $\theta=140^{\circ}$, definito in maniera arbitraria. Il trasduttore 1 è inclinato in modo da favorire la ricezione delle onde che viaggiano in verso orario, mentre il trasduttore 2 (inclinato dello stesso angolo) favorisce la ricezione delle onde antiorarie. Il disco viene ispezionato per una profondità di $1.9 \mathrm{~mm}$, pari alla lunghezza d'onda dell'onda superficiale data dal rapporto tra la velocità dell'onda superficiale nell'alluminio $\left(\mathrm{V}_{\mathrm{Al}}=2.9 \mathrm{~mm} / \mu \mathrm{s}\right)$ e la frequenza dell'onda $(1.5 \mathrm{MHz})$.

In condizioni statiche, in conseguenza alla loro disposizione simmetrica rispetto al punto di origine delle onde ultrasonore, i due trasduttori acquisiscono le stesse forme d'onda sul disco integro (Fig. 2). La prima onda (R1) ha il seguente tempo di volo (TOF):

$$
T \mathrm{OF}_{\mathrm{R} 1}=\frac{\mathrm{S}_{\mathrm{Al}}}{\mathrm{V}_{\mathrm{Al}}}+\frac{\mathrm{S}_{\text {aria }}}{\mathrm{V}_{\text {aria }}}=\frac{245.6}{2.9}+\frac{5.5}{0.33} \cong 101 \mu \mathrm{s}
$$

dove $\mathrm{S}_{\mathrm{Al}}$ ed $\mathrm{S}_{\text {aria }}$ sono la distanza percorsa nel disco e in aria, Varia la velocità dell'onda in aria. La seconda onda (R2), ovvero la prima che si presenta nuovamente al sensore dopo aver percorso tutta la circonferenza del disco, ha tempo di volo pari a:

$$
T \mathrm{OF}_{\mathrm{R} 2}=\frac{\mathrm{S}_{d}}{\mathrm{~V}_{\mathrm{A} 1}}+T \mathrm{OF}_{\mathrm{R} 1}=\frac{632}{2.9}+101 \cong 319 \mu \mathrm{s}
$$

dove con $S_{d}$ si è indicata la lunghezza della circonferenza del disco. Il tempo necessario affinché l'onda percorra un giro completo della superficie del disco è pari a $218 \mu \mathrm{s}$.

Inoltre, nel caso statico, si hanno fenomeni di interferenza delle due onde propaganti in senso opposto, caratterizzate da identici tempi di volo, tali da provocare aumenti dell'ampiezza delle stesse ad ogni mezza rivoluzione (rilevabile con un sensore posto a $180^{\circ}$ ).

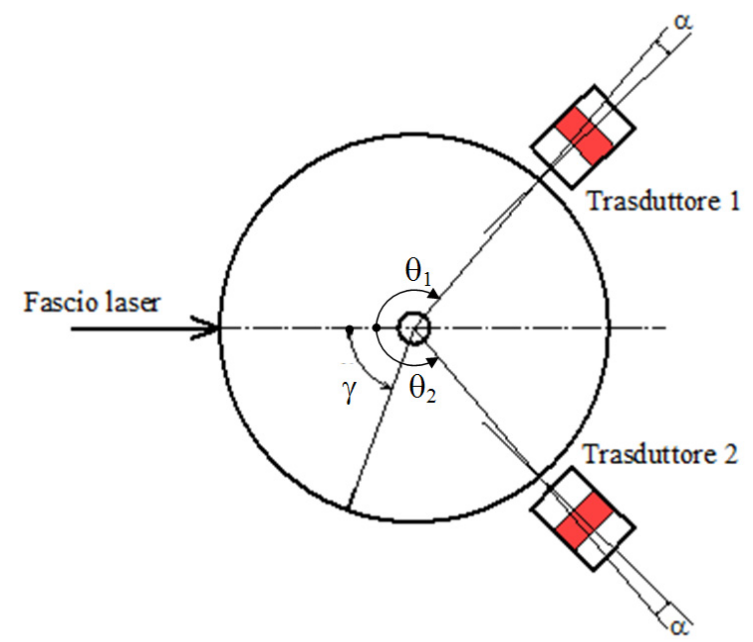

Figura 1: Configurazione per il rilievo di difetti superficiali con onda che si propaga in direzione circonferenziale. Figure 1: Setup to detect surface defects using the wave propagating along the circumferential direction. 

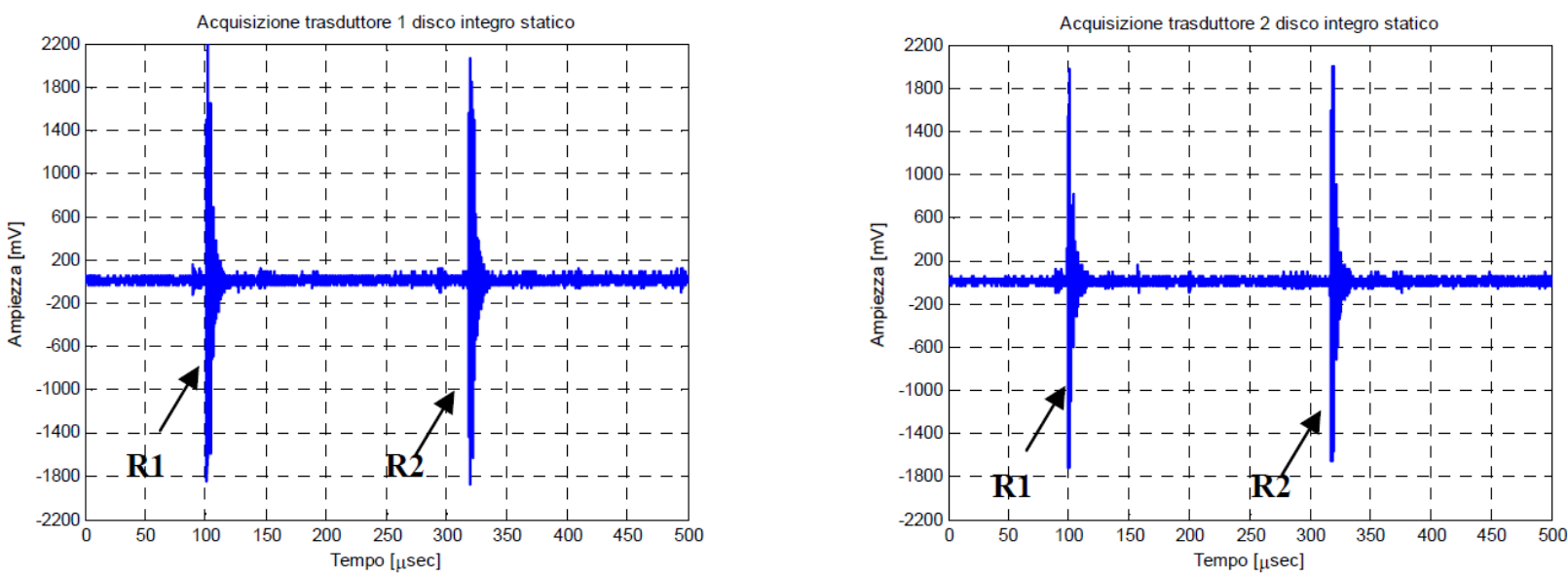

Figura 2: Segnale acquisito dai trasduttori 1 e 2 sul disco integro in condizioni statiche.

Figure 2: Signal acquired by transducers 1 and 2 in the pristine disk in static conditions..

Posto in rotazione il disco a 2800 giri/min, secondo verso antiorario in Fig. 1, sono stati acquisisti i segnali in condizioni dinamiche. La Fig. 3 confronta il segnale acquisito, sul disco integro, in condizione statica (blu) e dinamica (verde).
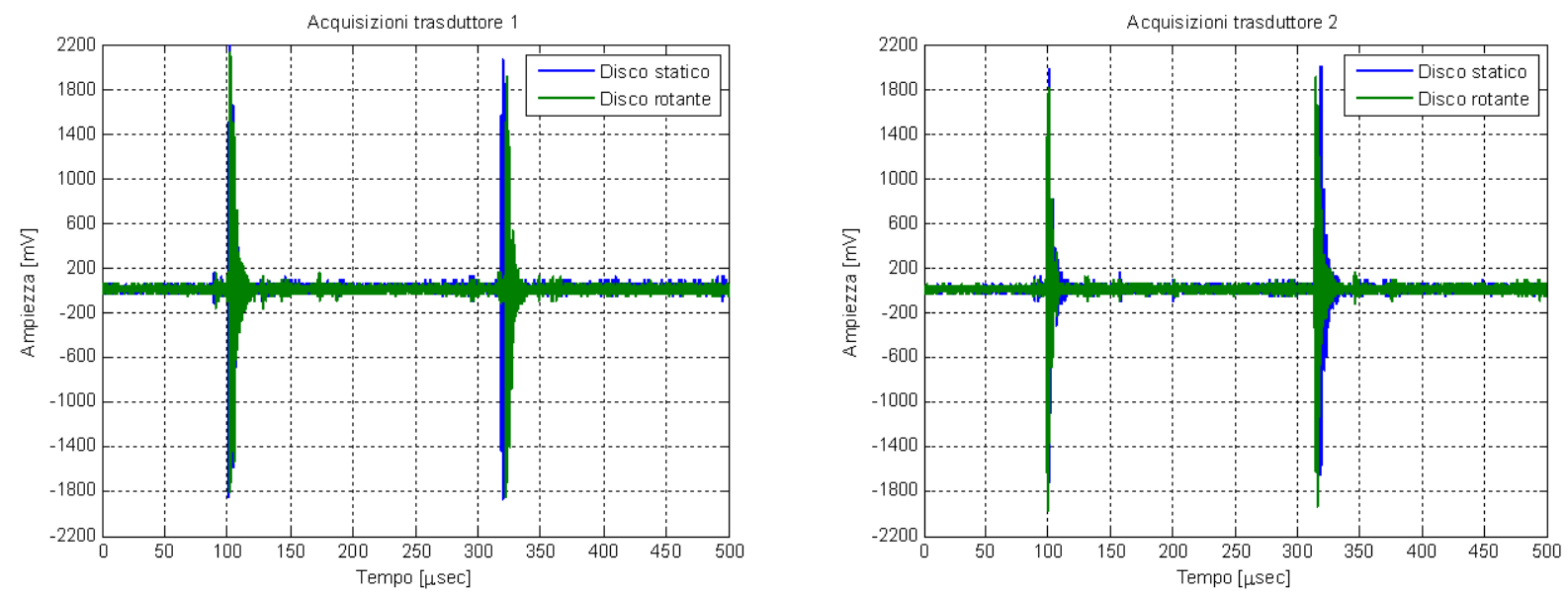

Figura 3: Confronto tra i segnali acquisiti dal trasduttore 1 e 2 in condizione statica e dinamica.

Figure 3: Comparison between the signals acquired by transducers 1 and 2 in static and dynamic conditions.

Come si evince dalla Fig. 3, in condizione dinamica si rileva un ritardo (anticipo) nei tempi di arrivo delle onde acquisite dal sensore 1 (sensore 2), proporzionale alla distanza percorsa dalle stesse.

I tempi di volo misurati per le onde R1 e R2 per il trasduttore 1 valgono $T O F_{R 1}=102.1 \mu \mathrm{s}$ e $T O F_{R 2}=322.2 \mu \mathrm{s}$. In condizione dinamica il tempo necessario affinché l'onda ultrasonora oraria percorra tutta la circonferenza del disco è pari a $220.1 \mu \mathrm{s}$. I valori dei tempi di volo relativi al trasduttore 2 sono $T O F_{R 1}=97.9 \mu \mathrm{s}$ e $T O F_{R 2}=313.6 \mu \mathrm{s}$. L'onda ultrasonora antioraria percorre la circonferenza del disco in $215.7 \mu$ s. Le misure di tali variazioni concordano con i valori ricavati dalle relazioni analitiche nel caso di onda superficiale che si propaga nello stesso verso di rotazione del disco e in verso opposto. L'interferenza delle due onde propaganti in senso opposto si presenta anche nel caso dinamico, ma non si ha la periodicità prima descritta in conseguenza della variazione dei tempi di volo delle due onde.

In conseguenza al moto relativo tra onda superficiale e corpo, si genera inoltre una variazione della frequenza del segnale, noto come effetto Doppler. Tale effetto è risultato del tutto trascurabile per la velocità considerata.

La stessa tipologia di prove è stata eseguita nel disco con difetto. Si è prima analizzato il provino in condizione statica, posizionando il difetto in diversi punti ed acquisendo il segnale in arrivo ai due trasduttori. In particolare, indicando con $\gamma$ l'anomalia del piano contenente il difetto (Fig. 1) e definendo positivi gli angoli in verso orario, sono stati acquisiti segnali 
ponendo il difetto in diverse posizioni corrispondenti a $\gamma= \pm 20^{\circ}, \pm 45^{\circ}, \pm 70^{\circ}, \pm 120^{\circ}, \pm 150^{\circ}, \pm 180^{\circ}$. Viene riportata solamente una delle configurazioni, con difetto posto a $\gamma=-70^{\circ}$.

Il segnale acquisito dal trasduttore 1 (Fig. 4) contiene quattro onde: la prima è l'onda diretta (R1) che, generata dal fascio laser incidente sul disco, si propaga in verso orario. La seconda è l'onda riflessa (TOF=185.7 $\mu$ s) generata dal difetto, ovvero l'onda che, generata dal laser, propaga inizialmente in senso antiorario e poi, in conseguenza alla riflessione in corrispondenza del difetto, prosegue verso il trasduttore propagando in senso orario. Le ultime due sono rispettivamente l'onda diretta (R2) e l'onda riflessa che, riuscendo ad oltrepassare il difetto, si ripresentano al trasduttore dopo aver percorso tutta la circonferenza del disco (in ritardo quindi rispetto alle prime di $218 \mu$ s). Queste ultime risultano notevolmente attenuate in conseguenza della trasmissione sul difetto.

Analoghi risultati si ottengono con riferimento ai segnali acquisiti dal trasduttore 2 (Fig. 5). In questo caso le onde chiaramente distinguibili sono quattro e relative, in sequenza d'arrivo, all'onda diretta che propaga in senso antiorario (R1) e riesce ad oltrepassare il difetto; all'onda che dopo aver percorso la superficie del disco in senso orario viene riflessa dal difetto e si propaga in senso antiorario verso il trasduttore ( $\mathrm{TOF}=234.6 \mu \mathrm{s})$. La terza è l'onda diretta $(\mathrm{R} 2)$ che oltrepassa nuovamente il difetto e si ripresenta in corrispondenza del trasduttore e, infine, l'onda riflessa dal difetto che compie l'intera rivoluzione, passando il difetto (TOF $=452.2 \mu \mathrm{s})$.

Mediante la valutazione dei tempi di volo dell'onda riflessa è possibile individuare univocamente la posizione del difetto, anche con un'unica ispezione. In presenza di più difetti lungo la circonferenza, il numero delle onde riflesse/trasmesse non permetterebbe più di individuare la posizione del difetto dal tempo di volo. In tal caso, si dovrebbe optare per una finestra temporale più stretta e più acquisizioni lungo la circonferenza. Ad ogni modo, per un'ispezione affidabile dovrebbero essere fatte almeno due acquisizioni a $180^{\circ}$ una dall'altra, per evitare che particolari posizioni del difetto (per esempio in corrispondenza della sorgente) diano segnali diversi.

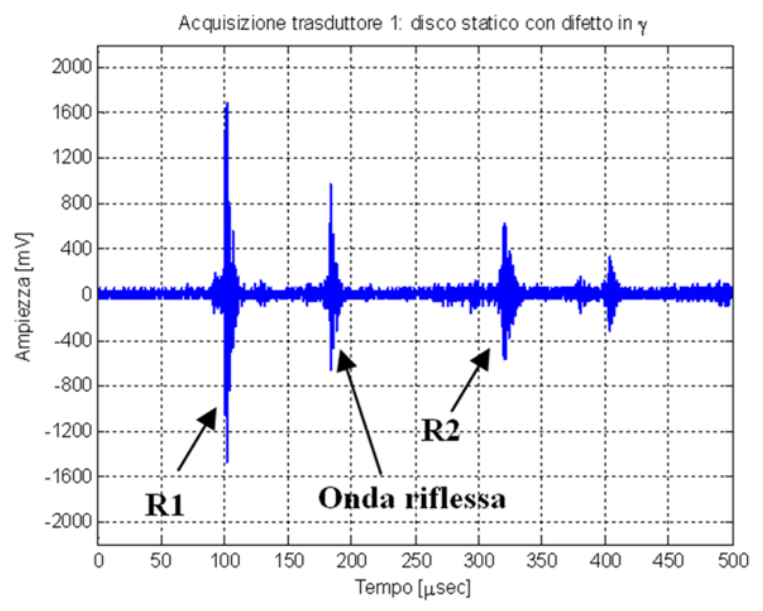

Figura 4: Segnale acquisito dal trasduttore 1. Disco fermo con difetto in $\gamma=-70^{\circ}$.

Figure 4: Signal acquired by transducer 1. Static disk with defect at $\gamma=-70^{\circ}$.

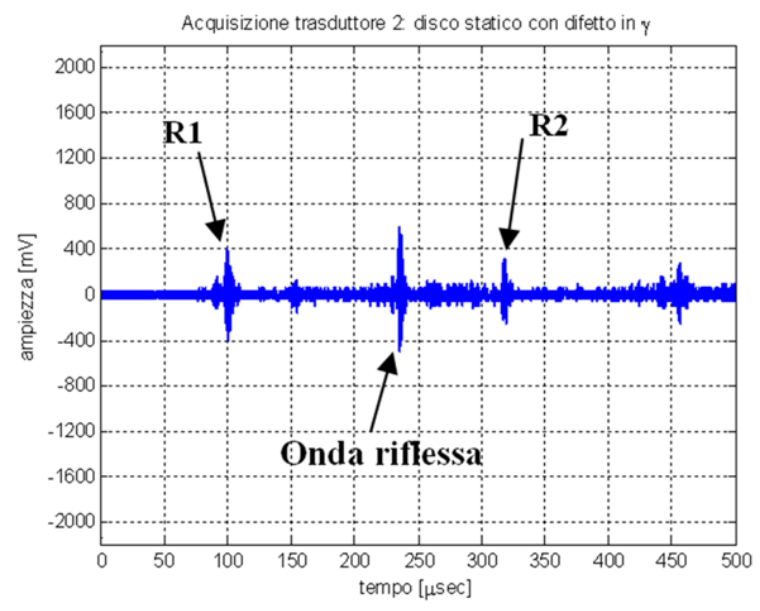

Figura 5: Segnale acquisito dal trasduttore 2. Disco fermo con difetto in $\gamma=-70^{\circ}$.

Figura 5: Signal acquired by transducer 2. Static disk with defect at $\gamma=-70^{\circ}$.

Dalle acquisizioni realizzate con il disco in rotazione si sono ottenuti segnali con onde riflesse caratterizzate da tempi di volo diversi. Ognuna di queste ha comunque permesso di individuare la presenza del difetto e di risalire alla sua posizione in quell'istante.

Per effettuare un confronto con l'acquisizione realizzata in condizioni statiche si riportano i segnali relativi alla posizione del difetto a $\gamma=-70^{\circ}$. Il calcolo dei tempi di volo delle onde R1 e R2 si esegue allo stesso modo, visto nel caso di disco senza difetto, in quanto esse propagano sempre in un verso. La determinazione dei tempi di volo delle onde riflesse risulta invece più complessa, in quanto la rotazione del disco tende a ritardarne l'arrivo nelle fasi in cui propagano in senso orario e ad anticiparlo nelle fasi in cui propagano in senso antiorario. Tenendo conto di tale aspetto si sono misurati $\mathrm{i}$ tempi di volo di tutte e quattro le onde, ottenendo una buona corrispondenza con i valori teorici (Fig. 6 e 7).

La seconda metodologia consiste nell'utilizzare due sensori, uno come emettitore e l'altro come ricevitore, in modalita pitch-catch, ispezionando con continuità la superficie del disco, lungo la generatrice (Fig. 8). L'onda analizzata è il fascio ultrasonoro riflesso dalla superficie del disco. 
I trasduttori sono di tipo capacitivo con frequenze emesse e rilevate nel range $0.1 \div 2.25 \mathrm{MHz}[15$, 16]. Entrambi i trasduttori sono stati disposti con assi giacenti su uno stesso piano, parallelo al piano orizzontale, ed inclinati simmetricamente rispetto al piano $\tau$ (normale all'asse del disco e passante per la mezzeria dello spessore) con angolo $\psi=45^{\circ}$, come mostrato schematicamente in Fig. 8. Il piano contenente gli assi dei trasduttori è stato inclinato di un angolo $\theta=45^{\circ}$, rispetto alla normale alla superficie del provino.

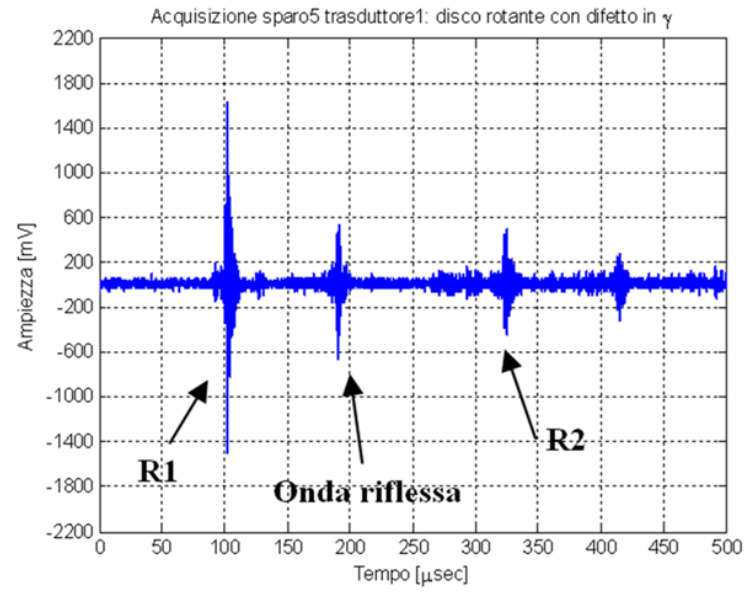

Figura 6: Segnale acquisito dal trasduttore 1. Disco rotante con difetto in $\gamma=-70^{\circ}$.

Figure 6: Signal acquired by transducer 1. Rotating disk with defect at $\gamma=-70^{\circ}$.

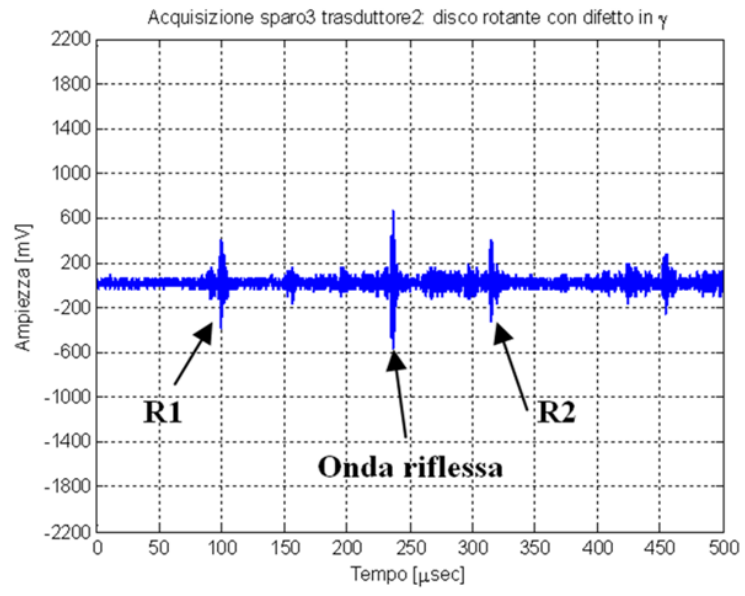

Figura 7: Segnale acquisito dal trasduttore 2. Disco rotante con difetto in $\gamma=-70^{\circ}$.

Figure 7: Signal acquired by transducer 2. Rotating disk with defect at $\gamma=-70^{\circ}$.
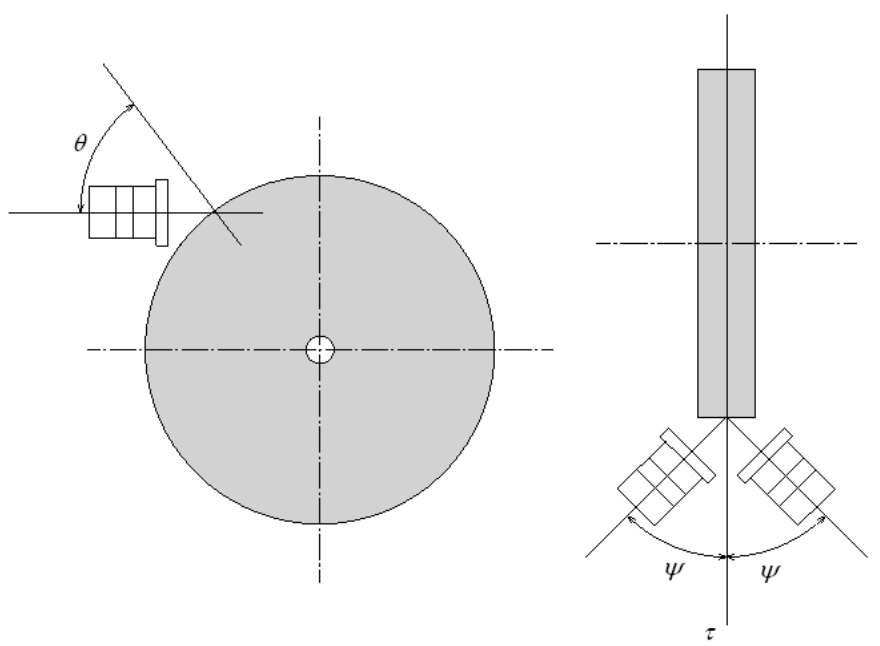

Figura 8: Configurazione per il rilievo di difetti superficiali con ispezione continua lungo la circonferenza del disco.

Figure 8: Setup to detect surface defects with continuous inspection along the circumference of the disc.

Per eseguire l'ispezione con continuità lungo la circonferenza del disco bisogna scegliere un generatore con frequenza di ripetizione opportuna; se la frequenza è minore si ha probabilità di non rilevare il difetto posto tra due impulsi consecutivi. A tal fine è necessario considerare la velocità di rotazione del disco, il diametro del disco e le dimensioni dell'area attiva dei trasduttori. Nel caso in esame la velocità periferica del disco è pari a $0.0294 \mathrm{~mm} / \mu$ s e la lunghezza della circonferenza del disco è pari a $\mathrm{S}_{\mathrm{d}}=632 \mathrm{~mm}$. Il tempo necessario affinché (in una rivoluzione del disco) il difetto si riporti nello stesso punto è quindi pari a $0.0215 \mathrm{~s}$. Avendo i trasduttori un'area sensibile con diametro di $10 \mathrm{~mm}$, per avere la certezza di ispezionare tutta la superficie del disco, è necessario avere un numero di impulsi $\mathrm{N}$ pari a 63.2. Per essere in grado di individuare il difetto in una rivoluzione del disco, è necessario avere $\mathrm{N}$ impulsi ogni 0.0215 secondi, ovvero 2939 impulsi al secondo. Il trasduttore emettitore è stato collegato ad un generatore con massimo valore della frequenza di ripetizione di $2 \mathrm{kHz}$. Con tale strumentazione si ha quindi una probabilità pari al 68\% di individuare il difetto in una rivoluzione del disco. 
Si sono quindi acquisiti i segnali sul disco in condizione statica in due distinte configurazioni: fascio ultrasonoro diretto su una porzione integra della superficie del disco e poi sul tratto di disco nel quale è presente il difetto. Si riportano in Fig. 9 e 10 i segnali ottenuti e le rispettive trasformate di Fourier.
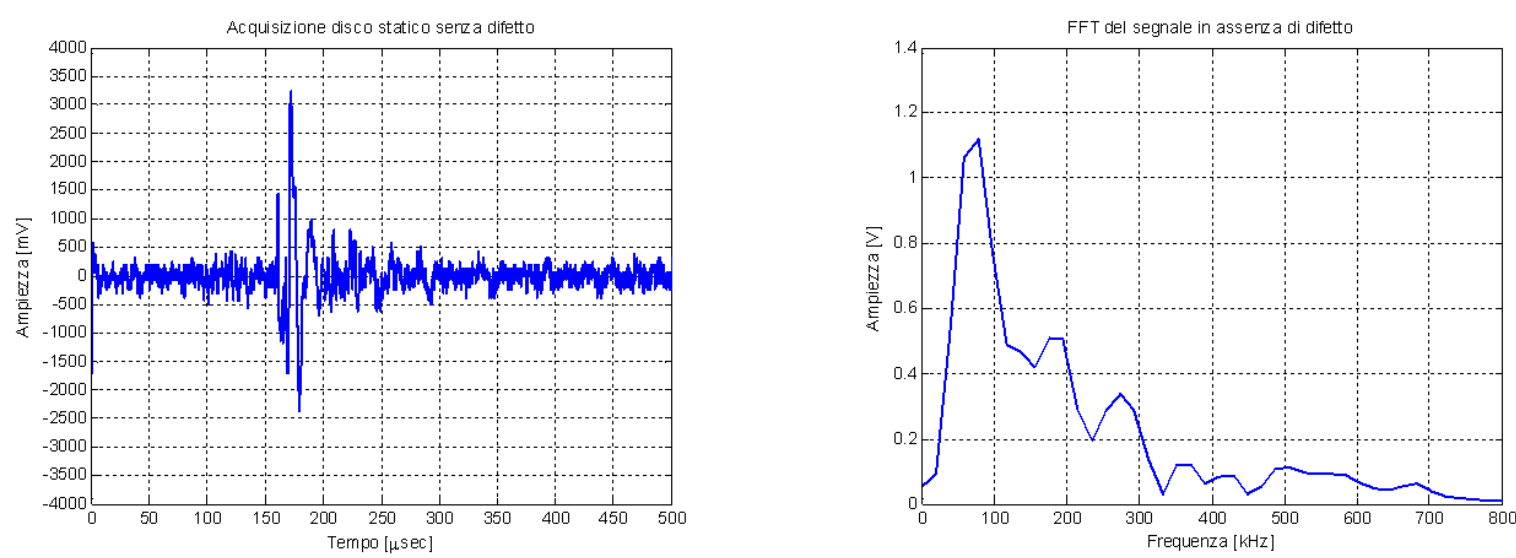

Figura 9. Segnale acquisito con disco fermo in assenza di difetto e relativa trasformata di Fourier. Figure 9. Signal acquired on the static disk, without defect, and its Fourier transform.
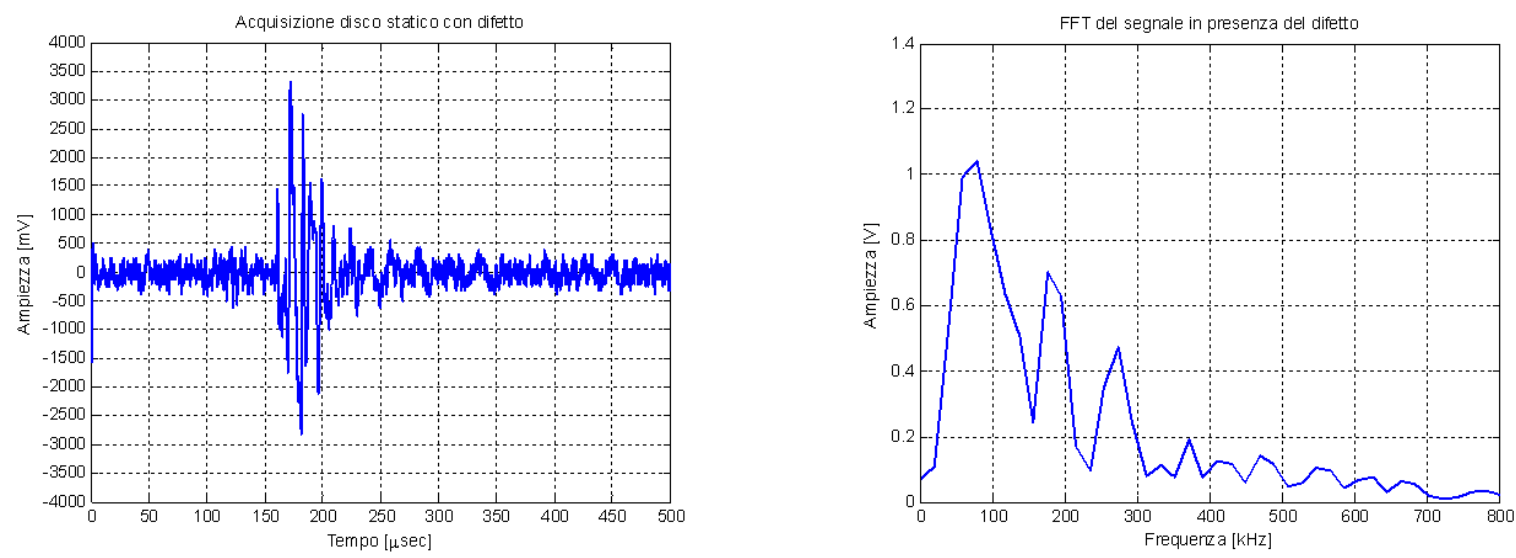

Figura 10: Segnale acquisito con disco fermo in presenza del difetto e relativa trasformata di Fourier.

Figure 10: Signal acquired on the static disk, with defect, and its Fourier transform.

Nel caso in cui il difetto si trova nella regione ispezionata dai trasduttori si ha una variazione della forma del segnale acquisito per la presenza delle onde diffratte ed una variazione del suo contenuto in frequenza. La nascita di onde diffratte, dovute alla discontinuità superficiale, produce infatti un aumento dell'ampiezza dei picchi nei campi di frequenza pari a $150 \div 200 \mathrm{kHz}$ e $250 \div 300 \mathrm{kHz}$.

Caratterizzato il provino in condizioni statiche, si sono acquisiti i segnali con il disco in rotazione. Si riportano in Fig. 11 e 12 i segnali acquisiti e le rispettive trasformate di Fourier rispettivamente per le acquisizioni in cui il fascio ultrasonoro ha inciso sul disco in rotazione in tratti di superficie integra e in corrispondenza del difetto.

Dal confronto con le acquisizioni relative al caso statico, nell'istante in cui il difetto si viene trovare in corrispondenza dei trasduttori si osserva una variazione del segnale nel dominio del tempo ed in quello della frequenza. Per il monitoraggio continuo, il criterio da utilizzare per discriminare in tempo reale $\mathrm{i}$ segnali acquisiti dalle aree integre da quelle con discontinuità è stabilire delle soglie per le ampiezze delle componenti di frequenza a $150 \div 200 \mathrm{kHz}$ e $250 \div 300 \mathrm{kHz}$ (ad esempio, rispettivamente, $0.6 \mathrm{~V}$ e $0.4 \mathrm{~V}$ per il caso in esame). L'informazione sulla posizione del difetto può essere fornita da un sensore angolare. Poiché l'indicazione di difetto viene data da onde che sono riflesse da discontinuità superficiale, allora elevata rugosità superficiale o scalfiture pronunciate possono influenzare il segnale. Pertanto le soglie dei picchi di frequenza devono essere calibrate sul difetto di interesse. Studi sistematici dovrebbero essere eseguiti per una valutazione quantitativa. 

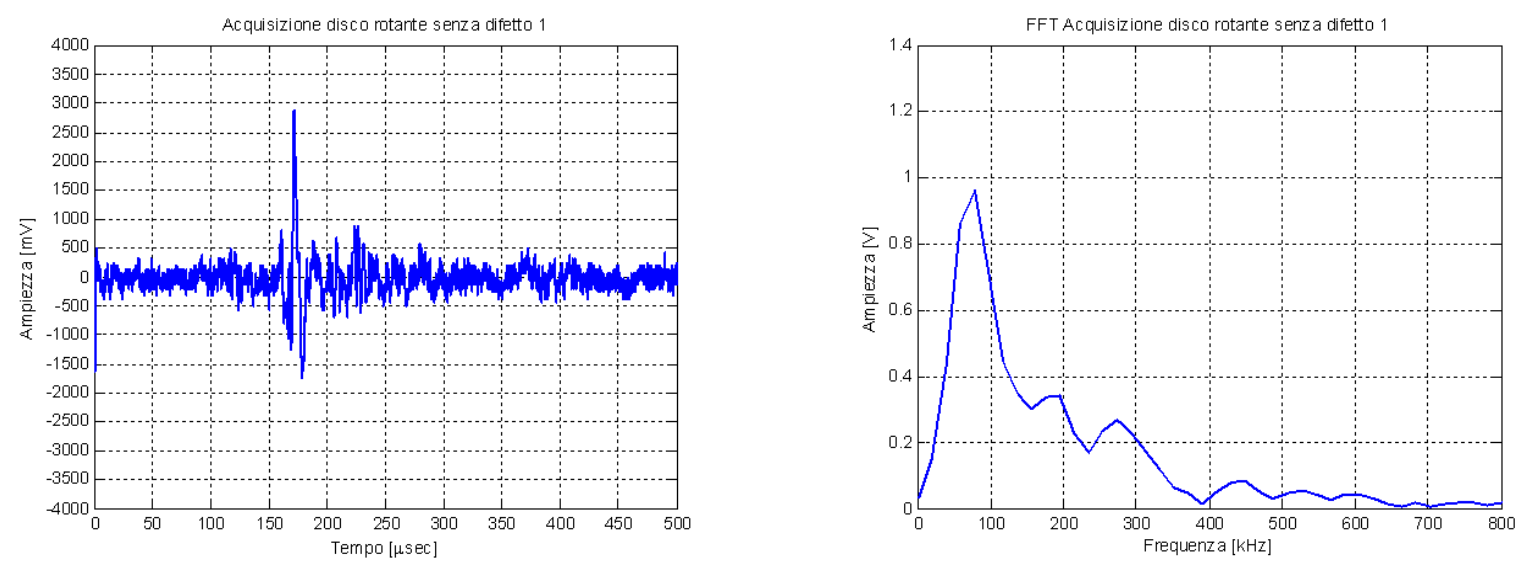

Figura 11: Segnale acquisito con disco rotante in assenza di difetto e relativa trasformata di Fourier.

Figure 11: Signal acquired on the rotating disk, without defect, and its Fourier transform.
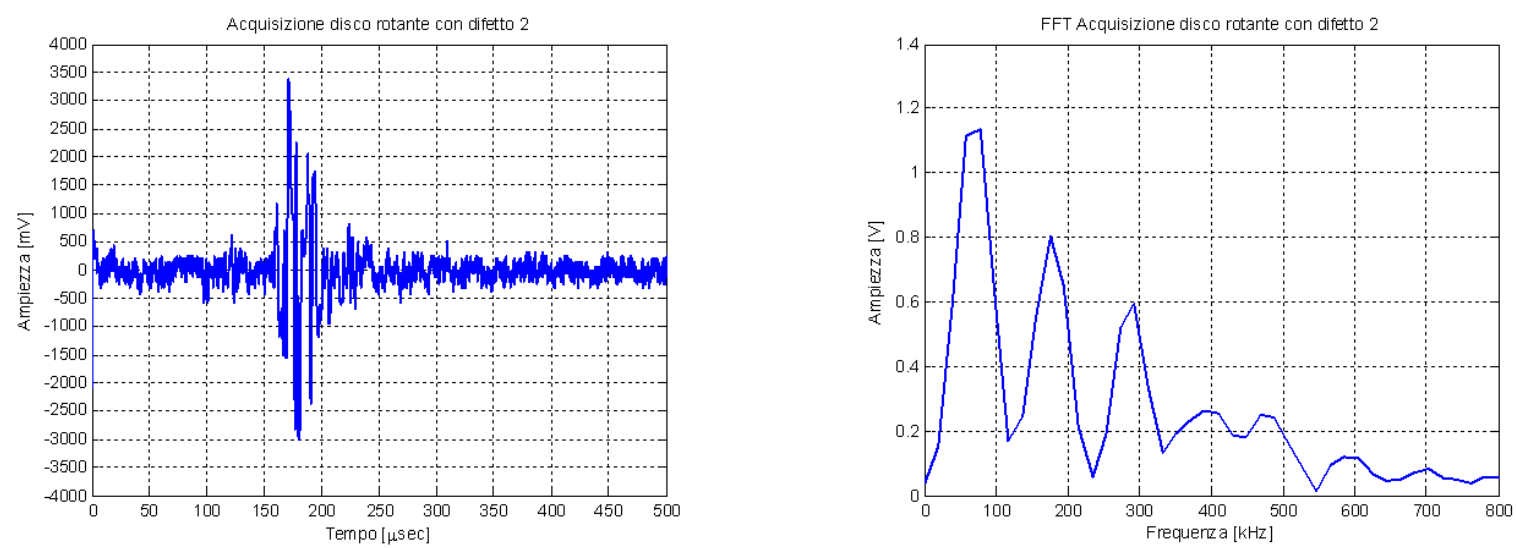

Figura 12: Segnale acquisito con disco rotante in presenza del difetto e relativa trasformata di Fourier. Figure 12: Signal acquired on the rotating disk, with defect, and its Fourier transform.

\section{CONCLUSIONE}

I

1 rilievo di difetti superficiali durante le ispezioni periodiche è importante poiché in genere le sollecitazioni sono maggiori in superficie e possono accelerare la crescita delle discontinuità. I metodi tradizionali di controllo non distruttivo, utilizzabili per i difetti superficiali, sono le correnti indotte, i liquidi penetranti, le polveri magnetiche e gli ultrasuoni. Alcuni tra questi metodi possono essere usati solo in condizioni statiche; gli altri presentano delle limitazioni per l'ispezione dinamica.

I progressi recenti nel campo dei sensori ultrasonori senza contatto ci hanno permesso di sviluppare un sistema semplice per il rilievo in tempo reale di difetti superficiali in corpi in movimento ad elevata velocità. Nel lavoro sono state presentate due metodologie di rilievo impiegabili col sistema sviluppato. Entrambe utilizzano onde ultrasonore, generate e rilevate con sonde senza contatto con la struttura. In una si sono utilizzati un laser pulsato e due trasduttori piezoelettrici, per generare ed analizzare l'onda superficiale che si propaga lungo la circonferenza, con una singola acquisizione. L'altra metodologia, sfruttando gli svantaggi della trasmissione degli ultrasuoni all'interfaccia aria/metallo, utilizza una coppia di trasduttori in modalità pitch-catch con ispezione continua lungo la circonferenza, valutando il fascio riflesso dalla superficie del disco.

Le metodologie sono state provate in laboratorio su due dischi in rotazione, di cui uno con difetto superficiale; entrambe si sono mostrate efficaci nell'individuazione del difetto in condizioni dinamiche, alla velocità di $100 \mathrm{~km} / \mathrm{h}$, con ottima ripetibilità dei risultati. L'esecuzione delle ispezioni risulta semplice con entrambe le tecniche. La preferenza di una all'altra è esclusivamente legata alla specifica applicazione. In un caso, il corpo viene ispezionato per una profondità pari alla lunghezza d'onda dell'onda superficiale generata; nell'altro l'sspezione è solo superficiale. Entrambe le metodologie 
permettono di rilevare in tempo reale e localizzare accuratamente la posizione del difetto. Con la configurazione sensore/sensore, per effettuare la scansione di tutta la superficie è necessario che la coppia di sensori si porti in corrispondenza di ogni suo punto, con ovvio aumento dei tempi d'ispezione rispetto alla configurazione laser/sensore. Tuttavia, la configurazione sensore/sensore permette di segnalare distintamente discontinuità multiple; tale attività diviene più complessa con l'altra configurazione.

\section{BIBLIOGRAFIA}

[1] M. C. Bhardwaj, Non-contact ultrasound: the last frontier in non-destructive testing and evaluation, Encyclopedia of Smart Materials, John Wiley \& Sons, New York, (2001).

[2] C.B. Scruby, L.E. Drain, Laser Ultrasonics: Techniques and Applications, Adam Hilger (1990).

[3] D.A. Hutchins, C.B. Scruby, R.J. Dewhurst, S.B. Palmer, J. Acoust. Soc. Am., 70(5) (1981) 1362.

[4] L.F. Bresse, D.A. Hutchins, J. Appl. Phys., 65(4) (1989) 1441.

[5] D. Cerniglia, A. Pantano, C. Mineo, Appl. Phys. A, Mater. Sci. Process., 105 (2011) 959.

[6] M. Arone, D. Cerniglia, V. Nigrelli, J. Mater. Process. Technol., 176 (2006) 95.

[7] D. Cerniglia, N. Montinaro, V. Nigrelli, J. Adhesion, 84 (2008) 811.

[8] A. Pantano, D. Cerniglia. Appl. Phys. A, Mater. Sci. Process., 91 (2008) 521.

[9] A. Pantano, D. Cerniglia. Appl. Phys. A, Mater. Sci. Process., 98 (2010) 327.

[10] I. A. Viktorov, Rayleigh and Lamb waves: Physical theory and applications (Plenum Press, New York, 1967).

[11] D. Clorennec, D. Royer. Appl. Phys. Lett., 82 (2003) 4608.

[12] S. Kenderian, B.B. Djordjevic, D.Cerniglia, G. Garcia. Insight 48, (2006) 336.

[13] D. Cerniglia, A. Pantano, M.A. Vento. J. Nondestruct. Eval., 31(3), (2012) 245.

[14] S. Kenderian, D. Cerniglia, B.B. Djordjevic, G. Garcia, J. Sun, M. Snell, Materials Evaluation, 61 (10) (2003) 1129.

[15] D.W. Schindel. Ultrasonics, 35 (1997) 179.

[16] W.M.D. Wright, D.W.Schindel, D.A. Hutchins. J. Acoust. Soc. Am., 95 (1994) 2567. 